\title{
Ferromagnetic and random spin ordering in diluted magnetic semiconductors
}

\author{
A. Kaminski, V.M. Galitski, and S. Das Sarma \\ Condensed Matter Theory Center, Department of Physics, \\ University of Maryland, College Park, Maryland 20742-4111
}

\begin{abstract}
In a diluted magnetic semiconductor system, the exchange interaction between magnetic impurities has two independent components: direct antiferromagnetic interaction and ferromagnetic interaction mediated by charge carriers. Depending on the system parameters, the ground state of the system may be ordered either ferromagnetically or randomly. In this paper we use percolation theory to find the ferromagnetic transition temperature and the location of the quantum critical point separating the ferromagnetic phase and a valence bond glass phase.
\end{abstract}

PACS numbers: 75.50.Pp, 75.10.-b, 75.30.Hx

\section{INTRODUCTION}

In diluted magnetic semiconductors (DMS), e.g. $\mathrm{Ga}_{1-x} \mathrm{Mn}_{x} \mathrm{As}, \mathrm{In}_{1-x} \mathrm{Mn}_{x} \mathrm{As}$ etc, long-range ferromagnetic ordering of the impurity (i.e. Mn) local moments is induced by the carriers (holes in most cases) which are contributed by the dopant impurities serving the dual role of acceptors and magnetic atoms. In principle, each Mn impurity atom contributes one hole, but heavy compensation intrinsically present in the system leads to a "dilute" carrier system with $n_{\mathrm{i}} \gg n_{\mathrm{h}}$, where $n_{\mathrm{i}}$ and $n_{\mathrm{h}}$ are the active local moment density and carrier density respectively. Throughout this paper, we refer to the carriers as "holes" and the magnetic local moments as "impurities" or "Mn atoms" without any loss of generality. The origin of this heavy compensation is essentially unknown although As antisite and Mn interstitial defects, both acting as electron doubledonors, are generally thought to be responsible. There is wide consensus that the ferromagnetic ordering of the impurity moments at low enough temperatures is caused by the local exchange interaction between the impurity atoms and the holes. This exchange coupling produces a long-range effective ferromagnetic interaction between the impurity local moments, leading to the ferromagnetic state of the system at temperatures $T<T_{c}$, where $T_{c}$ is the ferromagnetic transition temperature. The precise mechanism for the carrier-mediated magnetic coupling between the impurity atoms is still being discussed and debated in the literature, particularly for the metallic DMS systems where both free-carrier weak-coupling RKKY-Zener interaction ${ }^{1.2}$ and strong-coupling Zenerdouble-exchange interaction ${ }^{3}$ have been invoked depending on whether the holes reside in the semiconductor valence band or in the Mn-induced impurity band respectively. The issue of whether the carriers reside in the semiconductor valence band or in the impurity band in the semiconductor band gap has been controversial 4 The hole binding energy $(\sim 150 \mathrm{meV}$ above the valence band edge) contributed by $\mathrm{Mn}$ in GaMnAs is fairly deep in the band-gap, and therefore impurity band physics is certainly operational in the strongly localized insulating DMS regime where the physical situation must be of car- riers strongly bound to randomly localized magnetic impurities in the system. In our earlier publications ${ }^{5}$.6.7.8.9 we have developed a polaron percolation theory for DMS impurity ferromagnetism in the strongly localized insulating regime by showing that the localized holes produce magnetic clusters of bound magnetic polarons with the polaron size increasing with decreasing temperature until the magnetic clusters overlap through the whole sample leading to the ferromagnetic temperature-induced polaron percolation transition (more details on this scenario are provided in Sec. III). Such a magnetic polaron transition scenario has later been explicitly verified in direct numerical simulations of the DMS Hamiltonian. ${ }^{10}$ In the current work we generalize our polaron percolation picture by explicitly including in the theory the direct (i.e. not carrier-mediated) antiferromagnetic exchange interaction between the impurity atoms which play an important role in II-VI materials, where the direct antiferromagnetic exchange interaction is strong and at larger impurity concentrations in III-V materials, when the magnetic impurities are more likely to be at short distances from each other.

The DMS materials are interesting systems from the fundamental perspective of frustration physics because there are independent competing ferromagnetic and antiferromagnetic contributions to the magnetic Hamiltonian arising from totally distinct physical processes: the ferromagnetic contribution is carrier-mediated and effectively long-range whereas the antiferromagnetic contribution is extremely short-ranged direct Mn-Mn exchange. The disorder inherent in random locations of the impurity atoms (i.e. $\mathrm{Mn}$ in $\mathrm{Ga}_{1-x} \mathrm{Mn}_{x} \mathrm{As}$ ) coupled with the competing long-ranged (carrier-mediated) ferromagnetic and shortranged direct antiferromagnetic interaction among the impurity atoms leads to interesting frustration physics in the system. This frustration will eventually lead to a non-ferromagnetic ground state (possibly, a spin glass state) in the system at high enough impurity concentration and we study this DMS frustration physics within our polaron percolation model. We believe that this random competition between ferromagnetic and antiferromagnetic coupling leads to the disappearance of DMS ferromagnetism at high impurity concentration and is re- 
sponsible for the generic absence of ferromagnetism (with a few exceptions with very low $T_{c}$ values) in II-VI DMS materials. As described in the rest of this paper, we find that $T_{c}$ shows a maximum as a function of the magnetic impurity density $n_{\mathrm{i}}$ due to this random competition, and for large enough impurity density the system has a nonferromagnetic ground state. We would like to mention in this context Ref. 11, in which a spin glass state was observed in an insualting (Ga, Mn) $\mathrm{N}$ alloy (which is a III-V dilute magnetic semiconductor). It is possible that the spin glass freezing is due to the random antiferromagnetic coupling between magnetic impurities, which becomes dominant at high impurity concentration.

The outline of the paper is as follows: The model is introduced in Sec. III In Sec. III we describe the percolation theory approach we use to deal with the problem at hand. In Sec. IV we investigate suppression of the ferromagnetic transition temperature by direct antiferromagnetic interaction between the Mn impurities. The suppression is due to the fact that the coupling of spins to charge carriers and the strength of the interaction between spins strongly depend on the concentration of the magnetic atoms. We show that the approach used in Sec. IV fails at very large concentrations of Mn atoms. This case of large Mn concentrations is considered in Sec. $\mathbb{\nabla}$ where a different technique is used. The results of the paper are summarized in Sec. DI

\section{THE MODEL}

The magnetic Hamiltonian of the system has the form

$$
\hat{H}=\sum_{j k} J\left(r_{j k}\right) \mathbf{S}_{j} \mathbf{s}_{k}+\sum_{j_{1} j_{2}} J^{\mathrm{AF}}\left(r_{j_{1} j_{2}}\right) \mathbf{S}_{j_{1}} \mathbf{S}_{j_{2}},
$$

where indices $j$ and $k$ run over magnetic impurities and localized holes respectively, $\mathbf{S}_{j}$ and $\mathbf{s}_{k}$ denote spins of magnetic impurities and localized holes, $J\left(r_{j k}\right)$ is the constant of impurity-hole exchange interaction, with its dependence on the distance $r_{j k}$ between the hole localization center and the magnetic impurities given by

$$
J(r)=J_{0} \exp \left(-\frac{r}{L}\right),
$$

with $L$ being the hole localization radius. The precise origin for the hole localization is irrelevant for our theory - it could be Coulombic binding to the impurity acceptor or disorder-induced Anderson localization or any other relevant localization mechanism. The sign of the impurity-hole exchange interaction does not matter for the physical properties of the impurity subsystem, since the effective exchange interaction between impurities induced by the first term of Hamiltonian (11) is always ferromagnetic (see, for example, Ref. 5 ). We choose $J_{0}>0$ (that is antiferromagnetic) in our consideration without any loss of generality simply because that happens to be the case for the exchange coupling between a hole and a Mn moment in GaMnAs.

The direct antiferromagnetic interaction between magnetic impurities also decays exponentially with the distance,

$$
J^{\mathrm{AF}}(r)=J_{0}^{\mathrm{AF}} \exp \left(-\frac{r}{a}\right),
$$

and the corresponding decay length $a$ is much smaller than that of the impurity-hole interaction, $a \ll L$, since the former is determined by the (small) decay length of the electron wave function of an inner shell of the magnetic impurity, while the latter is determined by the (larger) decay length of the wave function of an impurityband carrier localized at some defect in the crystal structure.

Parameters of Hamiltonian (11), $J_{0}, J_{0}^{\mathrm{AF}}, a$, and $L$, together with impurity and hole concentrations $n_{\mathrm{i}}$ and $n_{\mathrm{h}}$ make the full set of parameters determining the properties of the system. In a realistic diluted magnetic semiconductor system, these parameters obey the following relations:

$$
\frac{1}{n_{\mathrm{h}}^{1 / 3}} \gg \frac{1}{n_{\mathrm{i}}^{1 / 3}} \gg a, \quad L \gg a .
$$

The fact that the charge carriers in the system under consideration are localized means that

$$
\frac{1}{n_{\mathrm{h}}^{1 / 3}} \gg L
$$

The relations between the pairs of parameters

$$
\frac{1}{n_{\mathrm{i}}^{1 / 3}} \text { and } L, \quad n_{\mathrm{h}}^{1 / 3} L \text { and } n_{\mathrm{i}}^{1 / 3} a
$$

are not predetermined in our consideration.

In this work, we will treat both impurity and hole spin dynamics classically. For impurity spins, it is justified by their relatively large value $S=5 / 2$ in real experimental systems, such as GaMnAs. The hole spins, even though not so large ( $s=3 / 2$ in GaMnAs), are strongly coupled to the magnetic impurities closest to the hole localization centers, which makes them "massive" (that is effectively classical) as far as their interaction with more distant impurities is concerned $\stackrel{5}{\underline{5}}$ In any case, the quantum properties are unlikely to be of qualitative importance in determining $T_{c}$.

For analytic description of the system described by Eqs. (11)-(4), it turns out to be important whether relation

$$
L \frac{1}{\left(L n_{\mathrm{h}}^{1 / 3}\right)^{1 / 3}} \gg \frac{1}{n_{\mathrm{i}}^{1 / 3}}
$$

is obeyed or not. The former case is more interesting physically, so it will be considered in this paper. The latter case requires a completely different formalism to treat but yields qualitatively similar results. In what follows we confine ourself to the former case, assuming that the inequality (6) holds. 


\section{PERCOLATION THEORY APPROACH}

\section{A. Basic principles}

To understand the physics of ferromagnetic transition in a diluted magnetic semiconductor system, it is easier first to consider the system at temperatures well above the ferromagnetic transition temperature, and then follow the evolution of the system as the temperature is lowered. So at high enough temperatures $\left(T \gg J_{0}\right)$ spins of holes and magnetic impurities are not correlated. As the temperature goes down, magnetic impurities close enough to hole localization centers tend to align their spins in the direction opposite to that of the hole they are close to. The resulting complex of a hole and magnetic impurities polarized by it is called a "bound magnetic polaron." 12 The radius of a bound magnetic polaron grows as $T \rightarrow 0$. When two or more bound magnetic polarons overlap, their spins must have the same direction. These overlapping polarons make polaron clusters; the ferromagnetic transition occurs when a macroscopic "infinite cluster" appears. In our earlier works ${ }^{5.9}$ we considered this ferromagnetic transition in the absence of antiferromagnetic interaction between magnetic impurities $\left(J_{0}^{\mathrm{AF}}=0\right)$. While this antiferromagnetic interaction certainly suppresses ferromagnetic transition in the system (to what extent, we will see later), the basic mechanics of the transition remains the same: bound magnetic polarons grow, merge, make polaron clusters, and finally formation of an infinite cluster signals ferromagnetic transition. We note that the DMS magnetic polaron percolation transition is not the usual concentrationdriven $T=0$ percolation transition, but a more subtle temperature-driven transition involving the coalescence of magnetic polaron clusters.

Thus a bound magnetic polaron is the basic unit of the mechanism of ferromagnetic transition in diluted magnetic semiconductors with localized charge carriers. We demonstrated in our earlier paper ${ }^{-}$that in the absence of antiferromagnetic interaction between the impurities they can be integrated out provided Eq. (6) holds, and the effective energy of the interaction between two polarons with distance $r$ between their centers can be obtained:

$$
\begin{aligned}
\left.\mathcal{E}_{\text {eff }}(r, \cos \theta, T)\right|_{J_{0}^{\mathrm{AF}}=0}=- & \frac{2 \pi}{3}\left[L^{2} r n_{\mathrm{i}} \ln \left(\frac{T}{J_{0}} e^{\frac{r}{2 L}}\right)\right] \\
& \times \frac{J_{0}^{2}}{T} \exp \left(-\frac{r}{L}\right) \cos \theta
\end{aligned}
$$

where $\theta$ is the angle between the spins of two polarons. One can see that the interaction between the polarons decays exponentially with distance between them. This reduces the problem of ferromagnetic transition in a system of bound magnetic polarons to the analogous problem of randomly placed spins with ferromagnetic exchange interaction between them decaying exponentially.

The latter problem was considered by Korenblit et al $\stackrel{13}{\underline{n}}$ in the context of ferromagnetic phase transitions in di- luted magnetic alloys. They proposed mapping of the physical problem at hand to the mathematical problem of percolation transition of same-radius randomly-placed spheres. Such a mapping is possible because of two factors: the exponentially fast decay of interaction between the spins and the characteristic distance between spins being much larger than the decay length of the interaction [see Eq. [4b]). Under these conditions, the distribution of the nearest-neighbor couplings is wide. This, in turn, means, that an any given temperature $T$ only a small fraction of nearest-neighbor-coupling strengths is of the order of $T$, and all the others are either much stronger or much weaker. The basic idea of the approach of Korenblit et al $l^{13}$ is that we can postulate all spins with coupling strengths between them stronger that $T$ to be locked in the same direction, and completely neglect all the other couplings, which, in general, are exponentially weaker. The justification for this approach is that there are very few couplings of the order of temperature, and their influence on the general picture is negligible; the validity of this justification will be discussed in the next subsection.

The formal mapping to the problem of percolating spheres is done in the following way: ${ }^{13}$ all spins are replaced with spheres of the same radius $R$, which is half the distance between two spins whose coupling exactly equals temperature. When two spheres (i.e spins) are closer than $2 R$, they overlap (and the coupling between the corresponding spins is larger than $T$ ). The spheres that overlap make clusters (which are magnetic clusters discussed above), and the ferromagnetic transition temperature is reached when the sphere radius (which grows as the temperature goes down) becomes large enough for the infinite cluster to appear. This problem of overlapping spheres has only one parameter, $n R^{3}$, where $n$ is the sphere concentration, and can be easily solved numerically. Mapping the results back to the original physical problem of interacting spins, we get expressions for the ferromagnetic transition temperature $T_{c}$ and other physical characteristics such as magnetization, susceptibility etc ${ }^{8.14}$ In the case of the system described by Hamiltonian (11) with $J_{0}^{\mathrm{AF}}=0$ the transition temperature is given by $\underline{5.9}$

$$
T_{c} \approx s S\left|J_{0}\right|\left(L n_{h}^{1 / 3}\right) \sqrt{n_{i} / n_{h}} \exp \left(-\frac{0.86}{L n_{h}^{1 / 3}}\right)
$$

We note that the solution of the problem involves two distinct mappings: first, the mapping of the DMS bound polaron Hamiltonian of holes and impurities to the problem of random spins with ferromagnetic exchange, and then the random spin problem to the mathematical percolation problem of random spheres. We also note that there is no characteristic temperature other than $T_{c}$ in this scenario. 


\section{B. On the applicability of the percolation picture}

In this section we discuss the limits of applicability of the percolation theory to the ferromagnetic transition in strongly disordered systems. Despite being an extremely useful tool in dealing with strongly disordered systems (sometimes, the only tool applicable), the percolation theory, as applied to temperature-driven phase transitions, has its drawbacks. Probably, the most important drawback, which is the center of this subsection's discussion, is the inability of the percolation theory to account for thermal fluctuations. We would like to emphasize that this drawback is not specific to the percolation of bound magnetic polarons, which we consider in this and our earlier papers $\frac{5,8.9}{1 n}$ fact, any treatment of the temperature-driven ferromagnetic phase transition in a strongly disordered using the percolation theory, including the paper by Korenblit et al. 13 in which this treatment was first proposed, inevitably neglects thermal fluctuations. In this section we argue that despite this problem, the ferromagnetic transition temperature, as predicted by the percolation theory, differs from the real transition temperature only by a numerical factor of the order of unity.

We argue that the percolation picture of the temperature-driven ferromagnetic phase transition in a strongly disordered system of spins is qualitatively correct. When the temperature goes down, ferromagnetic correlation is inevitably established first between the most-strongly-coupled spins, then spreading across weaker couplings. In such a system above the ferromagnetic transition temperature, one has ferromagnetically ordered regions (ferromagnetic clusters) without strong correlation between the magnetic moments of different clusters. As the temperature goes down, more couplings become saturated, so the new clusters appear and the existing ones grow and merge. Clearly at some low enough temperature this growth and merging of clusters will result in appearance of a correlated region which spans the whole sample (infinite cluster). This scenario is referred to, for example, in the context of the Griffiths phase physics, which was introduced before the first application of the percolation theory to the problem of ferromagnetic phase transitions. It seems to be universally accepted as far as the qualitative description of the physics is concerned. We have recently discussed the relevance of Griffiths phase to DMS systems in the context of the magnetic percolation transition in Ref. 9.

However, it is hard to build a rigorous controllable approach based on the above scenario. A reasonable approximation ${ }^{13}$ described in Sec. IIIA is to assume that at any given temperature $T$ two spins connected by some exchange coupling $J$ are completely uncorrelated if $J<T$, and locked in the same direction is $J>T$. Clearly, thermal fluctuations are completely left out of this approach. Despite this fact, such an approach should be adequate far from the transition point, when the clusters formed by the interacting spins have finite size. Any problems arising in estimating $T_{c}$ can be taken care of by introducing numeric corrections of the order of unity to the physical quantities calculated. The infinite cluster is, however, a different matter. By definition, spins making the infinite cluster must all be correlated across arbitrarily large distances. Small but finite deviations from the strict alignment may accumulate over large distances resulting in a complete loss of long-range coherence. Still one should agree that the exponent in Eq. (8) is predicted by the percolation theory correctly. Indeed, there can be no ferromagnetic order at $T \gg T_{c}$ with $T_{c}$ given by Eq. (8), because no connected network of couplings larger than such $T$ would exist. The connected network of such couplings does appear at $T \sim T_{c}$. The transition temperature, however, is determined not only by the strengths of the couplings making the connected network, but also by the topology of the network itself. The latter, however, is characterized only by power (not exponential) dependence on the system parameters, as it follows from the scaling assumptions of the percolation theory ${ }^{15}$ Therefore the exponent in Eq. (8) is the only exponent which may enter the expression for the true $T_{c}$.

Now we are going to argue that not only the exponent, but also the parametric prefactor at the exponent for the true $T_{c}$ is correctly predicted by the percolation theory. To begin with, let us note that the relation between the parameter $p \equiv n_{\mathrm{i}}^{1 / 3} r(T)$ of the percolation theory problem and the temperature reads

$$
p=n_{\mathrm{h}}^{1 / 3} L \log \left(\frac{J_{0}}{T}\right),
$$

with $n_{\mathrm{h}}^{1 / 3} L \ll 1$ [see Eq. (4b)], and the critical value of $p$ being $p_{c} \approx 0.86$. In the vicinity of the transition point, $p \approx p_{c}$, we have

$$
\frac{\left|p-p_{c}\right|}{p_{c}} \approx \frac{n_{\mathrm{h}}^{1 / 3} L}{0.86} \frac{\left|T-T_{c}\right|}{T_{c}} \ll \frac{\left|T-T_{c}\right|}{T_{c}} .
$$

Therefore, at $\left|T-T_{c}\right| / T_{c} \sim 1$, that is away from the thermodynamic transition, we still have $\left|p-p_{c}\right| / p_{c} \sim$ $n_{\mathrm{h}}^{1 / 3} L \ll 1$, so the system is still near the percolation threshold as far as the cluster topology and the scaling relations of the percolation theory are concerned.

Now let us consider the system of spins at some temperature $T=A T_{c}$, where $A<1$ is a constant of the order of unity. Let us consider the spins making up the infinite cluster (in terms of the percolation theory) and argue that this infinite cluster must be in the ferromagnetic state at some value of $A$, which is not parametrically smaller than unity. As the basis for our arguments, we are going to use the "links, nodes and blobs" picture 15,16 describing the topology of the infinite cluster in the vicinity of the percolation transition, see Fig. 11. According to this picture, the infinite cluster is a network of "links" connecting "nodes", with some occasional "blobs" embedded into the links. The link length is proportional to $\left|p-p_{c}\right|$ to some power of the order of unity. One may argue that the transition temperature of such a network is 




FIG. 1: Structure of the infinite cluster (after Refs. [15] and [16]).

of the order of $J_{\text {link }}$, where $J_{\text {link }}$ is the effective exchange interaction between the nodes as it is transmitted by the link. The distribution of the couplings along the link is wide, the weakest coupling is exponentially small as compared to the average one, and the length of the link is large only as a power of the same parameter that enters the exponent. In such a system, it is the weakest coupling which gives the value of $J_{\text {link }}$, and therefore the critical temperature must be of the order of the weakest coupling in a link, which is of the order of $T_{c}$ as given by Eq. (8). Therefore, the "real" critical temperature may not be parametrically smaller that the one predicted by the percolation theory.

Now let us argue that the "real" critical temperature should not be parametrically larger than the percolation theory prediction. In principle, there is only one link of the strength of $J_{\text {link }}$ between two nodes, so if it was the only link at all, the ferromagnetic temperature would have to be of the order of $J_{\text {link }}$. Even though other links connecting the nodes are weaker, they may significantly increase the critical temperature provided there is a sufficient number of them. We, however, argue that the number of weaker links is not large enough to compensate for their weakness. Indeed, the number of weaker links connecting two nodes may be as large as at most some power of the scaling parameter $\left|p-p_{c}\right| / p_{c}$, which at $T \sim T_{c}$ is of the order of $n_{\mathrm{h}}^{1 / 3} L$, see Eq. (10). The link strength, on the other hand, is widely distributed due to the exponential decay of the interaction and the probability of parametrically many links having their strength of the order of $J_{\text {link }}$ is exponentially small, so the connection between two nodes is dominated by the strongest link connecting them, whose strength is $J_{\text {link }}$, and the critical temperature may not be parametrically higher than $J_{\text {link }}$.

This reasoning leads us to the conclusion that while the ferromagnetic transition in the system under consideration is not, rigorously speaking, a percolation transition, the result for $T_{c}$ given by the percolation theory may differ from the real transition temperature only by a factor of the order of unity. We do not make any claims that our reasoning is a rigorous proof of our statement, but we do state that it is coherent with the established notions of the percolation theory 15 while the contrary statement would conflict with these notions. The final answer may be given only by a numerical simulation, and we point out that the Monte-Carlo results $\frac{17}{17}$ on the ferromagnetic transition temperature in diluted magnetic semiconductors with localized charge carriers is in excellent agreement with our theory ${ }^{5}$, which is an indication that the reasoning of this section is correct.

\section{SUPPRESSION OF $T_{c}$}

Because the decay length $a$ of the inter-impurity antiferromagnetic interaction is much less than the characteristic distance $n_{\mathrm{i}}^{1 / 3}$ between them, see Eq. 4a), the distribution of the coupling strength (3) between neighboring impurities is wide. Assuming the positions of the impurities to be random and uncorrelated, one can easily calculate the probability $P(J)$ that antiferromagnetic coupling $J^{\mathrm{AF}}$ between an impurity and its nearest neighbor is smaller than $J$ :

$$
P(J)=\exp \left[-\frac{4}{3} \pi n_{\mathrm{i}} a^{3}\left(\ln \frac{J_{0}^{\mathrm{AF}}}{J}\right)^{3}\right] .
$$

It has been demonstrated ${ }^{5.9 .18}$ that ferromagnetic interaction between two bound magnetic polarons (separated by the distance $r$ ) occurs mostly due to weaklypolarized lens-shaped region between the polaron centers, with "thickness" $L \ln \left[\left(T / J_{0}\right) \exp (r / 2 L)\right]$ and "radius" $\sqrt{L r}$, see Ref. 5 9. In the expression (7) for the effective energy of the polaron interaction with $J_{0}^{\mathrm{AF}}=0$, the term in square brackets is essentially the number of magnetic impurities in this lens-shaped region, whose (weak) polarization by one polaron affects the other polaron and vice versa. Non-zero antiferromagnetic interaction suppresses this interaction in the following way: if the distance $R$ between two impurities in the lensshaped region is small enough, so that $J^{\mathrm{AF}}(R)>T$, the spins of these two impurities are locked in the opposite directions, which means that they are not affected by the polarons and drop out of the group of impurities transferring interaction between the two polarons. The probability that an impurity is not excluded from this interaction transfer equals $P(T)$ with function $P$ given by Eq. (11). Therefore, the effective energy of interaction of two bound magnetic polarons in the presence of (direct) antiferromagnetic interaction between magnetic impurities is given by Eq. (7) with an extra factor of $P(T)$ in the right-hand side, which accounts for the reduction of the number of impurities mediating interaction between the two polarons [factor in brackets in Eq. (7)].

Since the concentration of holes is relatively low, $n_{\mathrm{h}} L^{3} \ll 1$, the relative variations of $\mathcal{E}_{\text {eff }}(r, \cos \theta, T)$ for different pairs of neighboring polarons are very large. It enables us to use percolation theory to establish the temperature of ferromagnetic transition in the system, 
as described in Sec. III According to the percolation theory, 5.13 the ferromagnetic transition temperature $T_{c}$ is given by the condition

$$
\mathcal{E}_{\text {eff }}\left(0.86 n_{\mathrm{h}}^{\frac{1}{3}}, \cos \theta, T_{c}\right)=T_{c}
$$

For not very large $J_{0}^{\mathrm{AF}}$, Eq. (12) can be solved for $T_{c}$ self-consistently by iterations, which yields:

$$
\begin{aligned}
T_{c} & \sim s S\left|J_{0}\right|\left(L n_{h}^{1 / 3}\right) \sqrt{n_{i} / n_{h}} \exp \left(-\frac{0.86}{L n_{h}^{1 / 3}}\right) \\
& \times \exp \left(-\frac{4 \pi}{3} 0.86^{3} \frac{a^{3} n_{\mathrm{i}}}{L^{3} n_{h}}\right) .
\end{aligned}
$$

This expression for $T_{c}$ becomes invalid when the antiferromagnetic interaction between the impurities becomes so strong that most of them are locked that is when $a^{3} n_{\mathrm{i}} \gg L^{3} n_{h}$. The next section deals with this case.

\section{QUANTUM PHASE TRANSITION}

At some critical concentration of magnetic impurities, the Curie temperature is suppressed to zero by the direct antiferromagnetic interaction. This is a quantum phase transition point separating a ferromagnetic state and a disordered state in the system of magnetic impurities (with the density of magnetic impurities being the control parameter).

Let us briefly and qualitatively discuss the possible structure of the disordered state. It is reasonable to start with a model in which there are only randomly placed magnetic impurities with antiferromagnetic interaction (3) between them, and no holes. The classical ground state in the continuum model is not frustrated as the interaction between impurities is a well-defined (exponential) function of the distance and the latter is a random variable. In the continuum model, the probability of finding, for example, a cluster of three impurities located in the corners of an equilateral triangle is exactly zero. One can say that the strong Possionian disorder in the continuum model lifts the degeneracy of the classical grounds state.

At low temperatures, the quantum nature of the spins of magnetic impurities should reveal itself. To get an insight into the ground state of the system one can use the idea of the real-space renormalization group technique frequently used in the studies of low dimensional antiferromagnetic systems $\stackrel{19}{12}$ In this method, one systematically integrate out the strongest bonds gradually reducing the energy scale at each step of the decimation procedure. The strongest bonds are considered frozen into a singlet state. The locked impurity pairs are then removed from the system; however, the couplings between other spins get renormalized due to virtual triplet excitations across the strong bonds. The main assumption of the real-space renormalization group technique is that physical properties are determined by an effective low-energy distribution of couplings (which in some cases has a universal form essentially independent of the initial distribution). The averaged characteristics of the spin system (such as correlation functions) are determined by very rare events, corresponding to a fraction of spins locked into a singlet being separated by a very large distance. These rare bonds dominate long-range correlation functions. It is important to emphasize that typical properties of the system are drastically different from the averaged properties and essentially determined by the majority of spins locked into a singlet with their nearest neighbours (one also should keep in mind that Griffiths effects are much less pronounced in higher dimensions). Therefore, the quantum ground state of our system can be represented as a set of randomly oriented spin pairs (valence bonds) locked into singlets. Such a state can be called a valence bond glass. Using this term, we can conclude that by increasing the density of magnetic impurities in a DMS system, one may induce a phase transition from a ferromagnetic (low impurity concentration) into a valence bond glass state, in which the typical correlation function decays, at the lengths of the order of characteristic separation $n_{\mathrm{i}}^{-1 / 3}$ between the impurities. At larger scales, the local impurity spin orientation can be considered as a random variable.

The actual quantitative details for the quantum phase transition to the valence bond glass phase are beyond the scope of the current work, where we consider the (classical) competition between random ferromagnetic and antiferromagnetic couplings induced by disorder at high magnetic impurity concentrations. It is clear, however, that the $T_{c}$-suppression given in Eq. (13) leads to a glassy magnetic state for large values of $n_{i}$, and quantum effects are obviously important in the elucidation of this glassy state.

\section{A. Bound magnetic polaron in a random medium of magnetic impurities}

Now let us consider one hole localized among magnetic impurities, still at zero temperature. Closer to the hole localization center, its interaction with the impurities is stronger than the antiferromagnetic interaction between the impurities, so all impurity spins in the vicinity of the hole localization center are aligned in the same direction. Away from the hole localization center, its interaction with the impurities becomes weaker, and at some distance $R_{\mathrm{p}}$ the antiferromagnetic interaction between impurities prevails over their interaction with the hole. To describe this behavior quantitatively, we introduce $n_{\mathrm{f}}(r)$, which is the concentration of the impurities aligned by their interaction with the hole spin rather than by their antiferromagnetic interaction with neighboring impurities. $n_{\mathrm{f}}(r)$ equals the product of the impurity concentration $n_{\mathrm{i}}$ and the probability $P[J(r)]$ that the coupling of 
and impurity to its nearest neighbor is weaker than its coupling $J(r)$ to the hole. Using Eqs. (2) and (11), we arrive at

$$
n_{\mathrm{f}}(r)=n_{\mathrm{i}} \exp \left[-\frac{4}{3} \pi a^{3} n_{\mathrm{i}}\left(\frac{r}{L}\right)^{3}\right] .
$$

The characteristic length $R_{\mathrm{p}}$, at which $n_{\mathrm{f}}(r)$ decays is given by

$$
R_{\mathrm{p}}=\frac{L}{n_{\mathrm{i}}^{1 / 3} a}
$$

and will be called the "zero-temperature radius of a bound magnetic polaron."

The magnetic impurities surrounding a bound magnetic polaron have some preferred spin directions determined by their antiferromagnetic interaction with their neighbors. A bound magnetic polaron "feels" this as an effective magnetic field $\mathbf{B}_{\text {rand }}$, whose amplitude and direction are random and are determined by the (random) configuration of impurities around it. Now we will estimate the characteristic magnitude of this field. There are two random contributions to this field. One, $\mathbf{B}_{\text {rand }}^{(1)}$, comes from the interaction of the hole with the magnetic impurities which are not polarized by it. The other contribution, $\mathbf{B}_{\text {rand }}^{(2)}$, is due to the interaction of the impurities polarized by the hole with the unpolarized ones. The energy coming from interaction of an impurity spin polarized by the hole with other polarized impurities is also random, but it does not depend on the hole's spin orientation, so it should not be included into the effective magnetic field which represents the action of the random impurity medium on the hole spin.

Rigorous analytic evaluation of these two contributions is hardly possible. One could naively expect that they can be expressed in terms of integrals of $n_{\mathrm{f}}(r), n_{\mathrm{i}}-n_{\mathrm{f}}(r)$, and $J(r)$ over volume. However, while $n_{\mathrm{f}}(r)$ is indeed the concentration of impurities whose spin direction is determined by the hole spin, $n_{\mathrm{i}}-n_{\mathrm{f}}(r)$ is not the concentration of randomly oriented impurities that determine the magnitude and direction of $\mathbf{B}_{\text {rand }}$. The reason is that in addition to two above mentioned categories of impurities - the ones polarized by the hole and the ones belonging to the random medium which extends away from the holes - there is a third category. An example of impurities of this third category is given by two impurities at the distance $r_{*}<R_{\mathrm{p}}$ from the hole localization center with the distance between them small enough for the antiferromagnetic interaction between them to be stronger than $J\left(r_{*}\right)$. One can easily see that these two impurities do not belong to those making $n_{\mathrm{f}}(r)$, but since this pair is able to rotate as a whole it will orient itself in the most energetically favorable position with respect to the spin of the hole, it will not contribute to $\mathbf{B}_{\text {rand }}$. We are not aware of any analytic way to separate these impurities from the random continuum, and therefore we must limit ourselves to estimates of $\mathbf{B}_{\text {rand }}$ instead of a rigorous evaluation.
For this estimate, we simplify the picture to the following: we assume that all the impurities whose distance to the hole localization center is smaller than $R_{\mathrm{p}}$ have their spins set by the hole spin, and that all the impurities beyond $R_{\mathrm{p}}$ belong to the random continuum. In this "setup" the estimation for $\mathbf{B}_{\text {rand }}$ is straightforward and yields:

$$
\begin{array}{r}
B_{\text {rand }}^{(1)} \sim \sqrt{n_{\mathrm{i}} L R_{\mathrm{p}}^{2}} J_{0} \exp \left(-\frac{R_{\mathrm{p}}}{L}\right) \\
B_{\text {rand }}^{(2)} \sim n_{\mathrm{i}}^{1 / 3} R_{\mathrm{p}} J_{0}^{\mathrm{AF}} \exp \left(-\frac{1}{n_{\mathrm{i}}^{1 / 3} a}\right)
\end{array}
$$

In these equations, the prefactors should probably be disregarded since they are likely to be artifacts of the simplification made above. The exponent $J_{0} \exp \left(-R_{\mathrm{p}} / L\right) \equiv$ $J_{0}^{\mathrm{AF}} \exp \left(-1 / n_{\mathrm{i}}^{1 / 3} a\right)$, however, which is notably the same for both $B_{\text {rand }}^{(1)}$ and $B_{\text {rand }}^{(2)}$, is highly likely correct, so it will be taken as our estimate for $B_{\text {rand }}$. Since the method employed above was rather crude, we may not be sure that a numerical factor of the order of unity [similar to 0.86 in Eq. (8)] was not missed, so we write the equation for $B_{\text {rand }}$ in the following form:

$$
\ln \left(\frac{B_{\text {rand }}}{J_{0}^{\mathrm{AF}}}\right) \sim-\frac{1}{n_{\mathrm{i}}^{1 / 3} a}
$$

\section{B. Interaction of two polarons in random continuum}

Now, having considered interaction of one bound magnetic polaron with the random medium made by interacting magnetic impurities, we turn to interaction between two polarons surrounded by interacting magnetic impurities and separated by distance $r$ from each other. In Ref. 5 and Sec. IV] of this paper we evaluated the interaction strength between two bound magnetic polarons with high precision, that is up to a numerical prefactor. Such a precision would be excessive in the case considered in this section, since this interaction is to be compared with the interaction with the random medium surrounding polarons, for which only estimate (17) is available. Therefore we may limit ourselves to an estimate of the polaron interaction strength. The (ferromagnetic) interaction comes from the interaction of each polaron's hole with the magnetic impurities polarized by the other, with the concentration of the latter given by Eq. (14). The strength of this interaction reads:

$$
\mathcal{E}_{\mathrm{eff}}(r, \cos \theta, T) \sim-J_{0} \exp \left(-\frac{r}{L_{\mathrm{loc}}}\right) \cos \theta,
$$

with all dimensionless prefactors omitted due to the reasons outlined above. Comparing Eq. (18) with Eq. (17) we immediately come to a (natural) conclusion that two polarons at zero temperature will have their spins aligned 


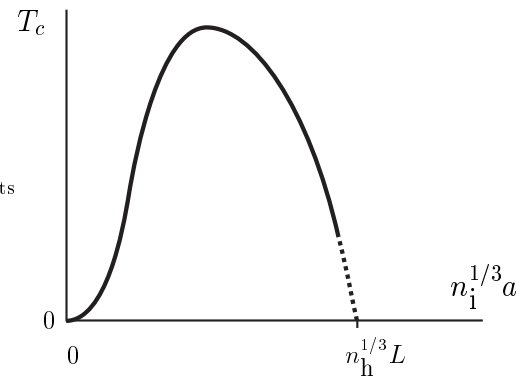

FIG. 2: Temperature of ferromagnetic transition $T_{c}$ as a function of dimensionless impurity concentration $n_{\mathrm{i}}^{1 / 3} a$, with $n_{\mathrm{h}}^{1 / 3} L=$ const. The solid line is determined by Eq. (13). The zero-temperature transition point is found in Sec. $\nabla$ The exact behavior of the dotted curve are beyond the scope of this paper.

in the same direction if the distance between them satisfies the condition

$$
r<2 R_{\mathrm{p}}
$$

where $R_{\mathrm{p}}$ is the zero-temperature polaron radius given by Eq. (15), and will have their spins pointing into two uncorrelated directions, which are determined by their interaction with the random medium of magnetic impurities if condition (19) does not hold.

Employing notions of the percolation theory we used above, we can use parameter

$$
\mathcal{P}=\frac{n_{\mathrm{h}}^{1 / 3} L}{n_{\mathrm{i}}^{1 / 3} a} .
$$

to characterize the ground state of the system. If $\mathcal{P} \gg 1$, the bound magnetic polarons form a ferromagneticallyordered infinite cluster, and the ground state of the system is ferromagnetic. If $\mathcal{P} \ll 1$, the interaction with the randomly-polarized medium of magnetic impurities breaks correlation between the cluster spins, and no longrange ordering of bound magnetic polarons is possible. The ground state of the system will be randomly ordered, with occasional isolated polarized regions around bound magnetic polarons and their finite-size clusters. Note that this statement is in agreement with result (13) of Sec. IV] which states that the ferromagnetic critical temperature becomes strongly suppressed (as compared to its value when $J_{0}^{\mathrm{AF}}=0$ ) exactly when $\mathcal{P} \approx 1$. We deliberately refrain from calling this random state a spin glass state since the words "spin glass" imply some very specific properties, which this randomly ordered state may not possess.

\section{CONCLUSION}

The results of this paper presented in Sec. IV and $\nabla$ can be summarized in Fig. [2] At very low concentration of magnetic impurities, the ferromagnetic transition temperature is low, because of the obvious reason that the impurity atoms mediate ferromagnetic interaction between bound magnetic polarons. Low concentration of impurities means weak interaction between polarons and, therefore, low transition temperature. As the concentration of impurities goes up, the ferromagnetic interaction between bound magnetic polarons becomes stronger, and $T_{c}$ increases. At lower concentration of magnetic impurities, antiferromagnetic interaction between them can be neglected, due to its small decay length $a$ [Eq. (3)], as compared to the large decay length $L$ of the impurityhole interaction. At a certain value of the concentration of magnetic impurities, this antiferromagnetic interaction becomes important and eventually the interaction between the polarons weakens, and $T_{c}$ goes down as $n_{\mathrm{i}}^{1 / 3} a$ grows, see Eq. (13). We note that such a non-monotonic dependence of the ferromagnetic transition temperature was observed in experiment [see, e. g. Ref. 20]. At a certain value of the concentration of magnetic impurities the Curie temperature is suppressed to zero and a quantum phase transition from a ferromagnetic state into a valence bond glass state is anticipated [see Sec. V]. The actual nature of this disordered valence bond phase remains an interesting topic for future theoretical studies.

We have also argued in this paper that, although the DMS ferromagnetic transition is strictly speaking not a percolation transition, the polaron percolation theory should provide a reasonable estimate for the ferromagnetic transition temperature $T_{c}$, see Sec. III

\section{Acknowledgments}

This work is supported by US-ONR and DARPA.
1 A. A. Abrikosov and L. P. Gorkov, Sov. Phys. JETP 16, 1575 (1963).

2 T. Dietl, H. Ohno, and F. Matsukura, Phys. Rev. B 63, 195205 (2001).

3 A. Chattopadhyay, S. Das Sarma, and A. J. Millis, Phys. Rev. Lett. 87, 227202 (2001).

4 C. Timm, J. Phys.: Condensed Matter 15, R1865 (2003).

5 A. Kaminski and S. Das Sarma, Phys. Rev. Lett. 88,
$247202(2002)$.

6 A. Kaminski and S. Das Sarma, Phys. Rev. B 68, 235210 (2003).

7 S. Das Sarma, E. Hwang, and A. Kaminski, Solid. State. Commun. 127, 99 (2003).

8 S. Das Sarma, E. Hwang, and A. Kaminski, Phys. Rev. B 67, 155201 (2003).

9 V. M. Galitski, A. Kaminski, and S. Das Sarma, Phys. 
Rev. Lett. 92, 117203 (2004).

10 M. Mayr, G. Alvarez, and E. Dagotto, Phys. Rev. B 65, 241202 (2002).

11 S. Dhar, O. Brandt, A. Trampert, K. J. Friedland, Y. J. Sun, and K. H. Ploog, Phys. Rev. B 67, 165205 (2003).

12 A. C. Durst, R. N. Bhatt, and P. A. Wolff, Phys. Rev. B 65, 235205 (2002).

13 I. Y. Korenblit, E. F. Shender, and B. I. Shklovskii, Phys. Lett. 46A, 275 (1973).

14 I. Y. Korenblit and E. F. Shender, Sov. Phys. Usp. 21, 832 (1978).

15 D. Stauffer and A. Aharony, Introduction to percolation theory (Taylor \& Francis, Philadelphia, 1994), 2nd ed.

16 B. I. Shklovskii and A. L. Efros, Electronic properties of Doped Semiconductors (Springer-Verlag, Berlin, 2001).

17 X. Wan and R. N. Bhatt, cond-mat/0009161.

18 P. A. Wolff, R. N. Bhatt, and A. C. Durst, J. Appl. Phys. 79, 5196 (1996).

19 S. K. Ma, C. Dasgupta, and C. K. Hu, Phys. Rev. Lett. 43, 1434 (1979); D. S. Fisher, Phys. Rev. B 50, 3799 (1994).

20 F. Matsukura, H. Ohno, A. Shen, and Y. Sugawara, Phys. Rev. B 57 (1998). 